\title{
Phenobarbital and neonatal seizures affect cerebral oxygen metabolism: a near-infrared spectroscopy study
}

\author{
Max D. Sokoloff', Melissa A. Plegue ${ }^{2}$, Ronald D. Chervin ${ }^{3}$, John D.E. Barks ${ }^{4}$ and Renée A. Shellhaas ${ }^{4}$
}

BACKGROUND: Near-infrared spectroscopy (NIRS) measures oxygen metabolism and is increasingly used for monitoring critically ill neonates. The implications of NIRS-recorded data in this population are poorly understood. We evaluated NIRS monitoring for neonates with seizures.

METHODS: In neonates monitored with video-electroencephalography, NIRS-measured cerebral regional oxygen saturation $\left(\mathrm{rSO}_{2}\right)$ and systemic $\mathrm{O}_{2}$ saturation were recorded every 5 s. Mean $\mathrm{rSO}_{2}$ was extracted for 1-h blocks before, during, and after phenobarbital doses. For each electrographic seizure, mean $\mathrm{rSO}_{2}$ was extracted for a period of three times the duration of the seizure before and after the ictal pattern, as well as during the seizure. Linear mixed models were developed to assess the impact of phenobarbital administration and of seizures on $\mathrm{rSO}_{2}$ and fractional tissue oxygen extraction.

RESULTS: For 20 neonates (estimated gestational age: $39.6 \pm 1.5 w \mathrm{w}), 61$ phenobarbital doses and 40 seizures were analyzed. Cerebral $\mathrm{rSO}_{2}$ rose $(P=0.005)$, and fractional tissue oxygen extraction declined ( $P=0.018$ ) with increasing phenobarbital doses. $\mathrm{rSO}_{2}$ declined during seizures, compared with baseline and postictal phases (baseline 81.2 vs. ictal 77.7 vs. postictal $79.4 ; P=0.004)$. Fractional tissue oxygen extraction was highest during seizures $(P=0.002)$.

CONCLUSIONS: Cerebral oxygen metabolism decreases after phenobarbital administration and increases during seizures. These small, but clear, changes in cerebral oxygen metabolism merit assessment for potential clinical impact.

$\mathbf{S}^{\mathrm{s}}$ eizures are common in neonates and often associated with adverse outcomes (1). Whether seizures themselves harm the developing brain or are simply manifestations of abnormal cerebral physiology remains an active debate. Animal data suggest that seizures amplify brain injury (2). Neonates with hypoxic-ischemic encephalopathy (HIE) and high seizure burden show more significant brain injury on magnetic resonance imaging and have worse outcomes than those who are seizurefree (3-5). High seizure burden in critically ill neonates and older children has also been independently associated with a higher probability and magnitude of neurological decline (6).
Long-term outcome studies of neonates with seizures demonstrate that a majority suffer significant neurodevelopmental abnormalities (7).

Phenobarbital is typically used as an initial treatment for neonatal seizures, despite incomplete efficacy (7-9), and concerns that this medication could induce abnormal neuronal apoptosis and cognitive impairment (10). Furthermore, the safety of loading dose administration in low-birth-weight and preterm neonates has been questioned, with currently recommended doses leading to higher than anticipated drug levels (11).

The gold standard for seizure diagnosis in neonates is electroencephalography (EEG) monitoring (12). Recently, additional cerebral monitoring has been advocated. Among clinically available noninvasive devices, near-infrared spectroscopy (NIRS) has been suggested as a potentially helpful instrument for monitoring brain functional integrity $(13,14)$. Disturbances in cerebral oxygen metabolism and blood flow, measured by a transcranial doppler, have been linked to electrographic seizures $(15,16)$. The NIRS has been shown to reflect cerebral blood flow (17), but its utility as a bedside monitor for infants at risk for neonatal seizures remains uncertain. We hypothesized that this device could provide data on the pathophysiology of neonatal seizures and consequences of their treatment. We aimed to assess the use of NIRS monitoring for neonatal seizures and to determine whether NIRS reveals physiological changes associated with doses of phenobarbital. We hypothesized that cerebral oxygen metabolism would peak during neonatal seizures. We further theorized that phenobarbital administration would be associated with a decline in brain tissue oxygen extraction.

\section{RESULTS}

There were 61 doses of phenobarbital administered to 20 patients (mean gestational age: $39.55 \pm 1.47 \mathrm{wk}$ ), with the majority of neonates receiving more than one dose. There were 40 individual seizures recorded on 11 of the patients. The seizure etiologies included HIE $(N=7)$, neonatal epilepsy syndromes $(N=5)$, arterial ischemic stroke $(N=2)$, sepsis $(N=2)$, benign neonatal seizures $(N=1)$, sinovenous thrombosis

${ }^{1}$ College of Literature, Science, and the Arts, University of Michigan, Ann Arbor, Michigan; ${ }^{2}$ Center for Statistical Consultation and Research, University of Michigan, Ann Arbor, Michigan; ${ }^{3}$ Department of Neurology, University of Michigan, Ann Arbor, Michigan; ${ }^{4}$ Department of Pediatrics \& Communicable Diseases, University of Michigan, Ann Arbor, Michigan. Correspondence: Renée A. Shellhaas (shellhaa@med.umich.edu)

Received 7 August 2014; accepted 22 December 2014; advance online publication 22 April 2015. doi:10.1038/pr.2015.64 
$(N=1)$, intracranial hemorrhage $(N=1)$, and uncertain etiol$\operatorname{ogy}(N=1)$. The demographic data are presented in Table 1 .

\section{Phenobarbital Models}

The phenobarbital doses ranged from 2.1 to $20.3 \mathrm{mg} / \mathrm{kg}$, with 49 maintenance and 12 bolus doses. The absolute changes in regional oxygen saturation $\left(\mathrm{rSO}_{2}\right)$ and fractional tissue oxygen extraction (FTOE) associated with phenobarbital dosing were small (Table 2). The estimated mean left-cerebral $\mathrm{rSO}_{2}$ was higher at baseline compared with the hour after phenobarbital

Table 1. Demographics of 20 neonates monitored with simultaneous conventional EEG and NIRS

\begin{tabular}{lc}
\hline Gender & $N(\%)$ \\
Male & $10(50.0)$ \\
Female & $10(50.0)$ \\
& Mean (SD) \\
Birth weight (g) & $3,308(481)$ \\
Gestational age (wk) & $39.6(1.5)$ \\
Phenobarbital dose (mg) & $17.4(13.8 ;$ range: $6.0-69.0)$ \\
Dose/birth weight (mg/kg) & $5.2(4.1 ;$ range: $2.1-20.3)$ \\
Seizure duration (s) & $120.0(180.4 ;$ range: $16.0-510.0)$ \\
Phenobarbital doses per patient & $N(\%)$ \\
1 & $5(25.0)$ \\
2 & $2(10.0)$ \\
3 & $6(30.0)$ \\
4 & $1(5.0)$ \\
5 & $6(30.0)$ \\
Seizures per patient & $N(\%)$ \\
0 & $9(45.0)$ \\
1 & $1(5.0)$ \\
4 & $2(10.0)$ \\
\hline & $5(25.0)$ \\
deviation. & $3(15.0)$ \\
\hline &
\end{tabular}

administration $(75.8 \pm 2.84$ vs. $74.9 \pm 2.80$; Bonferroni-adjusted $P=0.049)$. In these analyses, there was no change in MAP, systemic $\mathrm{O}_{2}$ saturation $\left(\mathrm{SaO}_{2}\right)$, or systemic $\mathrm{rSO}_{2}$ associated with phenobarbital dosing.

The models refit to include an interaction between time period (1-h before, during, and after the dose) and weightadjusted doses $(\mathrm{mg} / \mathrm{kg})$ were significant. For bolus doses, the cerebral $\mathrm{rSO}_{2}$ was significantly higher in the hour after the dose, compared with the predose baseline $(83.4 \pm 2.4$ vs. $79.3 \pm 3.5, P=0.005$; Figure 1a), while $\mathrm{rSO}_{2}$ was lower in the hour after maintenance doses $(81.1 \pm 1.69$ vs. $82.9 \pm 1.53, P=$ 0.012 ). The cerebral $\mathrm{rSO}_{2}$ during the hour of phenobarbital administration did not differ significantly from baseline levels for either maintenance or bolus doses.

Systemic $\mathrm{O}_{2}$ saturation $\left(\mathrm{SaO}_{2}\right)$ was slightly higher during the hour of phenobarbital bolus dose administration, compared with baseline $(96.9 \pm 0.51 \%$ vs. $95.4 \pm 0.77 \%, P=0.014)$, and returned to baseline in the subsequent hour. The maintenance doses were not associated with significant changes in $\mathrm{SaO}_{2}$.

The models of FTOE, as derived from $\mathrm{rSO}_{2}$ and $\mathrm{SaO}_{2}$, predicted reduced cerebral FTOE, by $3.6 \%$ on average, after phenobarbital bolus dose administration, compared with baseline $(13.5 \pm 2.66 \%$ vs. $15.81 \pm 3.68 \%, P=0.018$; Figure $1 \mathbf{b})$. There was no significant difference in FTOE associated with maintenance doses.

\section{Seizure Models}

For data that included all 40 seizures, both right- and leftcerebral $\mathrm{rSO}_{2}$ declined during seizures, compared with baseline and postictal phases (right: $81.2 \pm 0.98$ vs. $77.7 \pm 2.57$ vs. $79.4 \pm 3.28, P=0.005$; left: $77.6 \pm 1.35$ vs. $74.9 \pm 1.96$ vs. $75.8 \pm 2.79, P=0.004$; Table 3 ). The right and left FTOE were highest during seizures (right: $23.7 \pm 1.64$ vs. $26.9 \pm 2.81$ vs. $23.9 \pm 2.91, P=0.002$; left: $19.9 \pm 1.90$ vs. $22.6 \pm 2.27$ vs. $20.0 \pm 3.19, P=0.002)$, compared with the baseline and the postictal periods (Table 3 ). There was no change in $\mathrm{SaO}_{2}$ or systemic NIRS variables ( $\mathrm{rSO}_{2}$ or FTOE) associated with seizures. The duration of seizures did not affect the results.

The model diagnostics demonstrated one subject to be highly influential in many of the results, based on Cook's distance

Table 2. Phenobarbital administration model results, controlling for dose ${ }^{a}$

\begin{tabular}{|c|c|c|c|c|}
\hline Outcome: average & $\begin{array}{l}\text { Predose, estimated } \\
\text { mean }(95 \% \mathrm{Cl})\end{array}$ & $\begin{array}{c}\text { During dose, estimated } \\
\text { mean }(95 \% \mathrm{Cl})\end{array}$ & $\begin{array}{l}\text { After dose, estimated } \\
\text { mean }(95 \% \mathrm{Cl})\end{array}$ & $P$-value \\
\hline Right-cerebral rSO $2_{2^{\prime}} n=56$ & $79.7(74.4,85.0)$ & $79.4(74.1,84.7)$ & $79.0(73.7,84.3)$ & 0.57 \\
\hline Left-cerebral rsO ${ }_{2}, n=59$ & $75.8(69.1,82.5)$ & $75.1(68.4,81.7)$ & $74.9(68.2,81.6)$ & 0.04 \\
\hline Systemic $\mathrm{rSO}_{2}, n=61$ & $77.8(74.0,81.6)$ & $77.6(73.8,81.4)$ & $79.5(75.7,83.3)$ & 0.16 \\
\hline $\mathrm{SaO}_{2^{\prime}} n=61$ & $96.7(95.9,97.6)$ & $96.7(95.8,97.5)$ & $96.4(95.6,97.3)$ & 0.52 \\
\hline MAP, $n=54$ & $53.5(49.8,57.2)$ & $54.3(50.6,58.0)$ & $53.1(49.4,56.8)$ & 0.22 \\
\hline Left-cerebral FTOE, $n=59$ & $22.0(15.0,28.9)$ & $22.5(15.6,29.4)$ & $22.3(15.3,29.2)$ & 0.42 \\
\hline Right-cerebral FTOE, $n=56$ & $17.8(12.2,23.3)$ & $18.0(12.5,23.6)$ & $18.0(12.4,23.5)$ & 0.91 \\
\hline Systemic FTOE, $n=61$ & $19.6(15.7,23.5)$ & $19.7(15.8,23.6)$ & $17.5(13.6,21.4)$ & 0.10 \\
\hline
\end{tabular}

$\mathrm{Cl}$, confidence interval; $\mathrm{FTOE}$, fractional tissue oxygen extraction; $\mathrm{rSO}_{2}$, regional oxygen saturation; $\mathrm{SaO}_{2}$, systemic $\mathrm{O}_{2}$ saturation.

astimated marginal means from linear mixed models with random intercepts for patient and dose event with ratio of dose to birth weight ( $\mathrm{mg} / \mathrm{kg}$ ) set to the average value of 5.24 $(\mathrm{mg} / \mathrm{kg}) . P$-value is from an overall Wald $\chi^{2}$ test for inclusion of time as a predictor in the model. Sample size represents the number of dose events included in the model. 
a

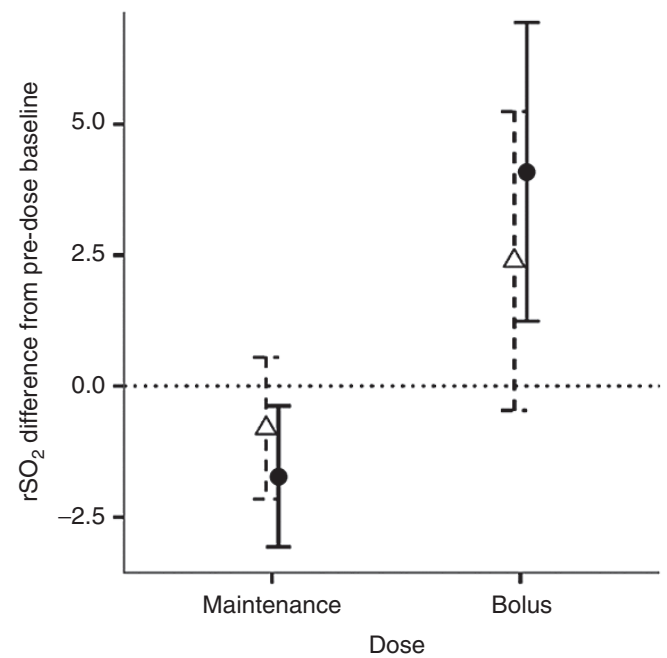

b

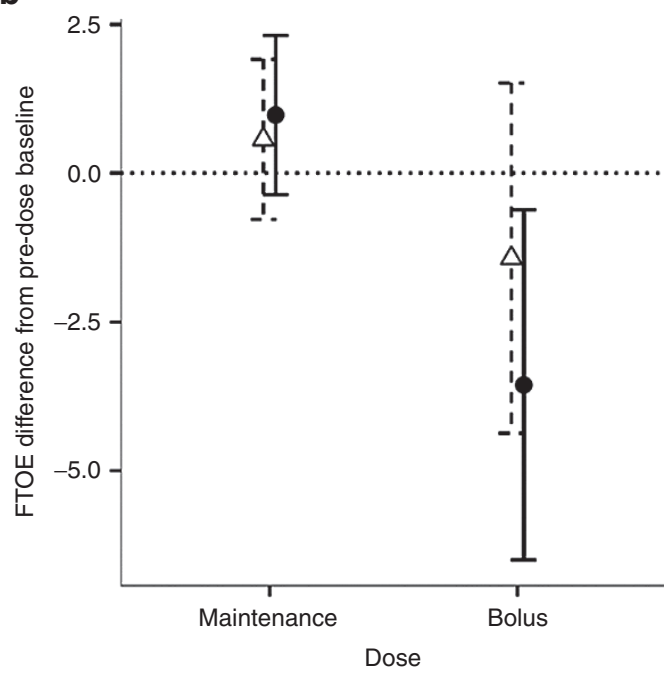

Figure 1. Changes in cerebral oxygen metabolism associated with phenobarbital administration. Linear mixed models with interactions were developed to assess the impact of phenobarbital administration on regional oxygen saturation $\left(\mathrm{rSO}_{2}\right)(\mathbf{a})$ and fractional tissue oxygen extraction (FTOE) (b). The estimated marginal means and SEs are presented for the difference in $\mathrm{rSO}_{2}$ and FTOE values measured at baseline vs. during phenobarbital dose administration (white triangles), and at baseline vs. $1 \mathrm{~h}$ after phenobarbital maintenance $(<10 \mathrm{mg} / \mathrm{kg}$ ) and bolus $(\geq 10 \mathrm{mg} / \mathrm{kg})$ doses (dark circles).

Table 3. Seizure model results ${ }^{\mathrm{a}}$

\begin{tabular}{|c|c|c|c|c|}
\hline & $\begin{array}{l}\text { Preseizure, estimated } \\
\text { mean }(95 \% \mathrm{Cl})\end{array}$ & $\begin{array}{l}\text { During seizure, } \\
\text { estimated mean }(95 \% \mathrm{Cl})\end{array}$ & $\begin{array}{l}\text { After seizure, estimated } \\
\text { mean }(95 \% \mathrm{Cl})\end{array}$ & $P$-value \\
\hline Right-cerebral rSO ${ }_{2^{\prime}} n=36$ & $81.2(72.0,90.3)$ & $77.7(68.5,86.8)$ & $79.4(70.3,88.6)$ & 0.005 \\
\hline Systemic rSO ${ }_{2}, n=40$ & $70.2(63.1,77.4)$ & $69.6(62.5,76.7)$ & $67.6(60.4,74.7)$ & 0.11 \\
\hline $\mathrm{SaO}_{2^{\prime}} n=40$ & $96.7(94.7,98.6)$ & $96.1(94.2,98.1)$ & $95.6(93.6,97.6)$ & 0.37 \\
\hline Right-cerebral FTOE, $n=37$ & $23.7(6.9,40.5)$ & $26.9(10.1,43.7)$ & $23.9(7.1,40.7)$ & 0.002 \\
\hline Systemic FTOE, $n=40$ & $27.2(20.1,34.3)$ & $27.9(20.8,35.0)$ & $28.6(21.6,35.7)$ & 0.51 \\
\hline
\end{tabular}

values. This infant's seizures were related to HIE, and the cerebral $\mathrm{rSO}_{2}$ declined more dramatically during seizures than the other subjects'. Removing this infant's data changed the statistical significance of the findings (Supplementary Table S1 online). The mean left-cerebral FTOE remained higher during seizures, compared with baseline (Bonferroni-adjusted $P=0.033$ ). The previously significant mean cerebral $\mathrm{rSO}_{2}$ rose (to $P=0.07-0.09$ ), just above the usual significance threshold.

\section{DISCUSSION}

This novel use of NIRS monitoring with concurrent conventional EEG to confirm neonatal seizures demonstrates that treatment with loading doses $(\geq 10 \mathrm{mg} / \mathrm{kg})$ of phenobarbital is associated with reduced cerebral oxygen metabolism (increased $\mathrm{rSO}_{2}$ and decreased FTOE) that persists for at least an hour after the dose is administered. EEG-confirmed neonatal seizures may, conversely, be associated with increased cerebral oxygen metabolism. The changes in oxygen metabolism were statistically robust, but the absolute differences in $\mathrm{rSO}_{2}$ were small and the $\mathrm{rSO}_{2}$ values always remained within the normal range. Thus, NIRS reveals compelling pathophysiological information, but assessment of potential clinical impact will require further study.

To our knowledge, we are the first to report changes in NIRS-measured cerebral oxygen metabolism associated with phenobarbital dosing for neonatal seizures. We found that the brain oxygen metabolism decreased after phenobarbital loading doses. This change was independent of seizure burden, since the seizures analyzed during this study were at separate times from the phenobarbital doses. Notably, the cerebral NIRS changes associated with phenobarbital and with seizures were also independent of systemic NIRS or MAP.

The published reports of NIRS changes associated with neonatal seizures have been confined to case studies. For a 39-wk male infant with HIE treated with phenobarbital and phenytoin, prolonged $(>7 \mathrm{~min})$ subclinical seizures were associated 


\section{Articles | sokoloffet al.}

with decreased cerebral tissue oxygenation index (analogous to $\left.\mathrm{rSO}_{2}\right)(14)$. This was similar to our finding of lower cerebral $\mathrm{rSO}_{2}$ values during seizures.

Two other case reports of infants monitored by NIRS and EEG demonstrated $\mathrm{rSO}_{2}$ fluctuations during seizures $(18,19)$. During a 30 -min epoch, the $\mathrm{rSO}_{2}$ of a 2 -mo-old preterm infant with cortical dysplasia abruptly declined 16 times, 11 of which were coincident with EEG-confirmed seizures (18). The authors noted that the NIRS patterns varied across individual seizures, a finding that is mirrored in our study, in which there was one individual whose seizure data were outliers. In another case study of a 2.5-mo-old infant with congenital heart disease, a repetitive spike pattern in NIRS-measured cerebral oxygenation was associated with subclinical status epilepticus (19). In all three case studies, and in our results, systemic oxygen saturation remained constant during the seizures.

None of the case studies reported FTOE changes associated with seizures or their treatment, but we showed that FTOE increased during seizures, even after an outlier was excluded. These findings echo previous work that described increased cerebral blood flow during electrographic and electroclinical neonatal seizures (15), and suggest that brain oxygen metabolism increases during neonatal seizures. Our data suggest that seizures place metabolic demands on the newborn brain. However, based solely on our results, we cannot be sure that these metabolic demands cause harm. The $\mathrm{rSO}_{2}$ was always within the expected physiological range and so the changes associated with seizures would not be expected to induce injury.

Few studies have differentiated between left- and rightcerebral NIRS measurements. In one study of very-preterm neonates, interhemispheric $\mathrm{rSO}_{2}$ and FTOE differences were noted during periods of unstable systemic oxygenation (20). In one of the above case studies, the right- and left-cerebral NIRS measures were recorded for a 30-min epoch. After the administration of pancuronium and subsequent doses of fosphenytoin, a decoupling of right- and left-cerebral NIRS data occurred (18). No physiological explanation was offered, only that the values did not appear to be artifact. Our study results also reflected differences between right- and left-cerebral NIRS measures, seen, e.g., in the mean $\mathrm{rSO}_{2}$ changes associated with phenobarbital across time (right: $P=0.57$ vs. left: $P=0.04$ ). The placement of NIRS sensors in clinical practice, on the forehead vs. over the parietal regions, may influence the test results and should be taken into consideration when data are interpreted.

Our study has some limitations. Although 20 infants were included, not all infants experienced seizures during the simultaneous NIRS-EEG monitoring. We attempted to avert erroneous conclusions related to outliers by limiting the data to a maximum of five seizures or phenobarbital doses per individual subject, and adjusting for subject in the models. There was one outlier subject in our dataset, who had substantial $\mathrm{rSO}_{2}$ changes during seizures. There were no obvious clues in the clinical or EEG data to suggest why that subject's NIRS patterns differed from the others. The subject was one of seven with HIE, and the seizure duration was not different from the others'. This subject's results dampened our conclusions about cerebral oxygen metabolism during seizures. However, the results also suggest that there may be some neonates for whom seizures can produce a clinically meaningful change in cerebral oxygen metabolism. We found no such outliers in the phenobarbital dosing analyses.

Although our findings regarding cerebral oxygen metabolism changes associated with phenobarbital loading doses are compelling, we only analyzed data from $1 \mathrm{~h}$ after medication administration. Thus, we are unable to comment on how long the documented changes in cerebral $\mathrm{rSO}_{2}$ were sustained. Also, we cannot comment on the effect of phenobarbital blood levels on NIRS results, since most infants did not have clinically indicated drug levels at the time of monitoring. However, higher doses of phenobarbital were associated with more significant changes in cerebral oxygen metabolism.

Our study subjects had seizures due to a range of etiologies. Due to the small subgroup sample sizes, we did not attempt to determine whether the NIRS changes associated with seizures or phenobarbital differed according to the underlying seizure etiology or gestational age. Additionally, we excluded moderate-preterm and very-preterm infants because their small head size precludes simultaneous monitoring of conventional EEG along with bilateral cerebral NIRS. Therefore, these data may not be directly applicable to one of the populations most commonly monitored with NIRS (16).

Most neonates in this study did not have invasive blood pressure monitoring. Artifact and missing data from noninvasive blood pressure measurements limited potential analysis. We are unable to comment on the possibility that abnormal cerebrovascular autoregulation could have contributed to the NIRS changes observed after phenobarbital administration. However, clinically significant hypotension was not noted for any of these infants.

There were significant strengths to this research. We provide real-world bedside physiological monitoring data, recorded with readily available clinical NIRS and EEG equipment, on late-preterm and term neonates with a variety of seizure etiologies. Our dataset, while limited, is notably larger than that of previous studies and provides insight for both the physiology of neonatal seizures and their treatment with phenobarbital. All analyzed seizures were identified using the gold standard multichannel conventional EEG. Since neonatal seizures are defined according to their EEG patterns, we did not classify seizures according to the presence or absence of associated clinical signs.

We utilized United States Food and Drug Administration (FDA)-approved neonatal NIRS sensors, which are the sensors most likely to be used in clinical practice in the United States. Others have reported NIRS data gathered with different sensor types. This methodological detail must be accounted for in data interpretation, since neonatal sensors' $\mathrm{rSO}_{2}$ results are typically higher than the pediatric sensors' results (21). We also studied $\mathrm{rSO}_{2}$ recorded over the parietal regions, rather than the typical frontal recording. This provided independent bihemispheric cerebral NIRS data, which were recorded from 
the cerebrovascular watershed regions and co-localized with the most common sites of origin for neonatal seizures (22). We also measured systemic $\mathrm{rSO}_{2}$, pulse oximetry, and, whenever possible, mean arterial blood pressure, in order to exclude changes in systemic physiology as causes for the cerebral oxygen metabolism variation associated with phenobarbital dosing and seizures. Notably, these systemic variables did not fluctuate with seizures or phenobarbital doses.

\section{Conclusions}

Typical phenobarbital loading doses are accompanied by prolonged declines in cerebral oxygen extraction, and neonatal seizures may be associated with transient increases in oxygen metabolism. Whether these small but statistically significant changes, measured by NIRS, have clinical consequences remains to be determined. Therefore, although NIRS may provide scientifically interesting data, our results alone do not provide rationale for routine clinical NIRS monitoring among neonates with seizures.

\section{METHODS}

The University of Michigan Institutional Review Board approved this study and the parent of each infant provided written informed consent. Each infant's chart was systematically reviewed for demographic and clinical details. Infants who required video-EEG monitoring due to clinical suspicion for seizures were included. Premature infants ( $\leq 35$-wk gestation) and those with multiple congenital anomalies were excluded. Infants were not excluded based on any other seizure etiology.

\section{Near-Infrared Spectroscopy (NIRS)}

The late-preterm and full-term neonates monitored with conventional video-EEG due to suspected seizures were simultaneously monitored with NIRS. The neonatal NIRS sensors (Invos 5100c, Somanetics Corp, Troy, MI) were placed over the bilateral parietal regions (the cerebrovascular watershed areas) and the thigh (systemic control). The NIRS-measured cerebral $\mathrm{rSO}_{2}$ and $\mathrm{SaO}_{2}$ (measured via pulse oximetry) were recorded every $5 \mathrm{~s}$ and downloaded to a research computer for post hoc analyses. The FTOE was calculated (FTOE $=$ $\left.\left(\mathrm{SaO}_{2}-\mathrm{rSO}_{2}\right) / \mathrm{SaO}_{2}\right)$.

\section{Phenobarbital}

The infants' medical records were systematically reviewed for phenobarbital dosing $(\mathrm{mg} / \mathrm{kg} /$ dose $)$ and administration data. A loading dose $(\geq 10 \mathrm{mg} / \mathrm{kg})$ of phenobarbital is typically administered over 20 min. The mean $\mathrm{rSO}_{2}$ was extracted for 1-h epochs before (baseline), during, and after each phenobarbital dose. To avoid erroneous conclusions based on overrepresentation of individual subjects, a maximum of five phenobarbital doses were analyzed per study subject, and statistical models took subject into account. The time periods for phenobarbital dose data did not overlap with the seizure data (see below). Linear mixed models with interactions were developed to assess the impact of phenobarbital administration on $\mathrm{rSO}_{2}$ and FTOE, adjusted for dose ( $\mathrm{mg} / \mathrm{kg})$.

\section{Seizures}

The electrographic seizures were defined as abnormal EEG events greater than $10 \mathrm{~s}$ in duration, with a clear evolution of frequency, morphology, and/or location, and a minimum of $2-\mu \mathrm{V}$ peak-to-peak amplitude (23). For each electrographic seizure, the mean and SD of $\mathrm{rSO}_{2}$ values were extracted for a period of three times the duration of the seizure before and after the ictal pattern, as well as during the seizure. This duration was selected in order to account for potential effects of variation in seizure duration (e.g., a prolonged seizure might result in a more prolonged postictal change in cerebral metabolism than a brief seizure). A maximum of five seizures were analyzed for each individual subject. The seizure times did not overlap with the above phenobarbital administration times. Linear mixed models and post hoc pairwise comparisons were employed to evaluate the seizure data, adjusted for seizure duration. Bonferroni-adjusted $P$-values were presented.

\section{Statistical Models}

Most neonates had more than one dose of phenobarbital or more than one seizure. Furthermore, each individual administration of medication or seizure produced three measurements, one for each time period (before, during, and after the seizure or phenobarbital dose). To account for this, the linear mixed models with random intercepts for patient and phenobarbital dose or seizure event were employed. The models were run separately for phenobarbital and seizure data, including weight-adjusted dosage $(\mathrm{mg} / \mathrm{kg})$ in phenobarbital models and length of seizure in seizure models as covariates. The significance of the effect of time period on the NIRS data was determined by a Wald $\chi^{2}$ test on the inclusion of time period coefficients in the models. Due to the small amount of missingness combined with the relatively small sample size, listwise deletion was used in the presence of missing data. All analyses were performed using Stata version 13.1 (StataCorp, College Station, TX).

To investigate whether weight-adjusted phenobarbital dose affected NIRS data, a second set of models was developed to include an interaction between time period and weight-adjusted dosage $(\mathrm{mg} / \mathrm{kg})$. The dose was categorized into bolus $(\geq 10 \mathrm{mg} / \mathrm{kg})$ or maintenance $(<10 \mathrm{mg} / \mathrm{kg})$ for a clinically meaningful comparison of time effect differences across dose values. The significance of interaction terms was determined through an overall Wald $\chi^{2}$ test. The effects of phenobarbital across the three time points were investigated through plots of estimated marginal means and differences between time periods for the categories of weight-adjusted doses (bolus vs. maintenance).

\section{SUPPLEMENTARY MATERIAL}

Supplementary material is linked to the online version of the paper at http:// www.nature.com/pr

\section{ACKNOWLEDGMENTS}

The near-infrared spectroscopy (NIRS) machines were donated by Somanetics Corporation (Troy, MI) for research use in our neonatal intensive care unit, but Somanetics had no input into study design, data analysis, writing of the manuscript, or the decision to submit this manuscript.

\section{STATEMENT OF FINANCIAL SUPPORT}

This research was supported by the National Institute of Child Health and Development (NICHD 5K23HD068402), the University of Michigan's Janette Ferrantino Investigator Award, and Charles Woodson Pediatric Biostatistics Fund (Ann Arbor, MI).

\section{REFERENCES}

1. Ramantani G. Neonatal epilepsy and underlying aetiology: to what extent do seizures and EEG abnormalities influence outcome? Epileptic Disord 2013;15:365-75.

2. Sampath D, White AM, Raol YH. Characterization of neonatal seizures in an animal model of hypoxic-ischemic encephalopathy. Epilepsia 2014;55:985-93.

3. Glass HC, Glidden D, Jeremy RJ, Barkovich AJ, Ferriero DM, Miller SP.Clinical Neonatal Seizures are Independently Associated with Outcome in Infants at Risk for Hypoxic-Ischemic Brain Injury. J Pediatr 2009;155:318-23.

4. Glass HC, Nash KB, Bonifacio SL, et al. Seizures and magnetic resonance imaging-detected brain injury in newborns cooled for hypoxic-ischemic encephalopathy. J Pediatr 2011;159:731-5.e1.

5. Wyatt JS, Gluckman PD, Liu PY, et al.; CoolCap Study Group. Determinants of outcomes after head cooling for neonatal encephalopathy. Pediatrics 2007;119:912-21.

6. Payne ET, Zhao XY, Frndova H, et al. Seizure burden is independently associated with short term outcome in critically ill children. Brain 2014;137(Pt 5):1429-38.

7. van der Heide MJ, Roze E, van der Veere CN, Ter Horst HJ, Brouwer OF, Bos AF. Long-term neurological outcome of term-born children treated 
with two or more anti-epileptic drugs during the neonatal period. Early Hum Dev 2012;88:33-8.

8. Painter MJ, Scher MS, Stein AD, et al. Phenobarbital compared with phenytoin for the treatment of neonatal seizures. N Engl J Med 1999;341:485-9.

9. Blume HK, Garrison MM, Christakis DA. Neonatal seizures: treatment and treatment variability in 31 United States pediatric hospitals. J Child Neurol 2009;24:148-54.

10. Bittigau P, Sifringer M, Genz K, et al. Antiepileptic drugs and apoptotic neurodegeneration in the developing brain. Proc Natl Acad Sci USA 2002;99:15089-94.

11. Oztekin O, Kalay S, Tezel G, Akcakus M, Oygur N. Can we safely administer the recommended dose of phenobarbital in very low birth weight infants? Childs Nerv Syst 2013;29:1353-7.

12. Shellhaas RA, Chang T, Tsuchida T, et al. The American Clinical Neurophysiology Society's Guideline on Continuous Electroencephalography Monitoring in Neonates. J Clin Neurophysiol 2011;28:611-7.

13. Lemmers PM, Zwanenburg RJ, Benders MJ, et al. Cerebral oxygenation and brain activity after perinatal asphyxia: does hypothermia change their prognostic value? Pediatr Res 2013;74:180-5.

14. Silas R, Sehgal A, Walker AM, Wong FY. Cerebral oxygenation during subclinical seizures in neonatal hypoxic-ischaemic encephalopathy. Eur J Paediatr Neurol 2012;16:304-7.

15. Boylan GB, Panerai RB, Rennie JM, Evans DH, Rabe-Hesketh S, Binnie CD. Cerebral blood flow velocity during neonatal seizures. Arch Dis Child Fetal Neonatal Ed 1999;80:F105-10.
16. Toet MC, Lemmers PM. Brain monitoring in neonates. Early Hum Dev 2009;85:77-84.

17. Tsuji M, duPlessis A, Taylor G, Crocker R, Volpe JJ. Near infrared spectroscopy detects cerebral ischemia during hypotension in piglets. Pediatr Res 1998;44:591-5.

18. Arca Diaz G, Cesaron E, Alfonso I, Dunoyer C, Yaylali I. Near infrared spectroscopy in the management of status epilepticus in a young infant. Eur J Paediatr Neurol 2006;10:19-21.

19. Giorni C, Di Chiara L, Cilio MR, et al. The usefulness of near-infrared spectroscopy for detecting and monitoring status epilepticus after pediatric cardiac surgery. J Cardiothorac Vasc Anesth 2009;23:668-71.

20. Lemmers PM, van Bel F. Left-to-right differences of regional cerebral oxygen saturation and oxygen extraction in preterm infants during the first days of life. Pediatr Res 2009;65:226-30.

21. Dix LM, van Bel F, Baerts W, Lemmers PM. Comparing near-infrared spectroscopy devices and their sensors for monitoring regional cerebral oxygen saturation in the neonate. Pediatr Res 2013;74:557-63.

22. Shellhaas RA, Clancy RR. Characterization of neonatal seizures by conventional EEG and single-channel EEG. Clin Neurophysiol 2007;118:2156-61.

23. Tsuchida TN, Wusthoff CJ, Shellhaas RA, et al.; American Clinical Neurophysiology Society Critical Care Monitoring Committee. American clinical neurophysiology society standardized EEG terminology and categorization for the description of continuous EEG monitoring in neonates: report of the American Clinical Neurophysiology Society critical care monitoring committee. J Clin Neurophysiol 2013;30:161-73. 